\title{
SIMULACIÓN Y OPTIMIZACIÓN EN LA LOGÍ́GTICA
}

\section{LUGISTICA}

PEDRO JDSÉ SANNCHEZ LAMAN*

\section{RESUMEN}

Actualmente, la investigación de operaciones es una herramienta útil en los proceso de diseño, ejecución y control de sistemas logísticos; sin embargo, una de las herramientas mas útiles es la simulación, la cual es una técnica asequible para la solución de problemas. Una de las primeras referencias en el tema de simulación se remonta al ano 1940 cuando el científico Ullman aplicó y diseño la técnica llamada "simulación Montecarlo". Más adelante la simulación inició una etapa de auge, mediante la aplicación en procesos en la industria aeroespacial, autopartista, de comunicaciones, entre otras; $y$ actualmente se utiliza en diversas áreas del sector empresarial y Militar, como es la logística, la producción, y últimamente en diversas actividades militares en todo el mundo, por ejemplo operaciones logísticas en la Guerra de Irak e Afganistan. El objetivo de este articulo es presentar una revisión crítica para quienes empiezan su investigación en el área de simulación de procesos logísticos y están interesados en la esta herramienta para mejorar la cadena de suministro en los niveles estratégico, táctico y operativo. En este articulo se presentará un marco teórico y conceptual que abordará la definición de simulación aplicada a la logística, sus principales aplicaciones, clasificación y metodología de desarrollo, información que intro- ducirá al lector en este tipo de procesos de optimización.

Palabras claves: simulación, cadenas de suministro, logística, optimización, investigación de operaciones

\section{ABSTRACT}

Nowadays, operations research is a useful tool in the process of design, execution and control of logistic systems; however, one of the most useful tools is simulation, which is an available technique for problem solving. One of the first references to simulation goes back to the year 1940, when a scientist by the name of UIIman, applied and designed the technique called "Montecarlo simulation". Simulation later began to grow through its application in NASA's aerospace design processes, among others, and at this moment simulation is used in diverse areas of the commercial, industrial, and military sectors, such as logistics, operation management, and lately in diverse military activities around the world, like the Iraq war. This article presents a critical overview for those who are beginning to research simulation in logistical processes and are interested in using this tool to improve strategic, tactical, and operative level supply chains. It defines

\footnotetext{
*Ingeniero Industrial de la Universidad Militar Nueva Granada, Especialista en Docencia Universitaria y Especialista en Gerencia en Logistica Integral. Docente de la Especialización en Logística del Instituto Militar Aeronáutico y de la Universidad Militar Nueva Granada en el programa de Ingeniería Industrial.
} 
simulation applied to logistics, its main applications, classifications, and development methodology, in order to introduce the reader to this kind of optimization processes.

El conocimiento transversal de la logística está dado por el diseño, modelamiento y aplicación de modelos matemáticos, concentrado especialmente en la investigación de operaciones, la cual surgió y adquirió su nombre durante la segunda guerra mundial y se consolidó durante la década de los 50. Sus primeras aplicaciones fueron en el campo militar y en el manejo y optimización de procesos logísticos militares. Desde entonces y hasta la actualidad el campo de aplicación fue creciendo e incluye numerosos problemas de la economía, la producción, la logística, empresas de servicios, organizaciones en general, y otras ciencias. Actualmente, la simulación es aplicada en la administración y diseño de sistemas logísticos como los siguientes: problemas de transporte, ruteo de vehícu- los, planificación de la producción, flujo en redes, análisis financiero de cadenas de abastecimiento, asignación de vehículos, y otros problemas como de la aplicación de herramientas metaheurísticas, optimización de la logística inversa, simulación de procesos, entre otros

La simulación ocupa un papel primordial en el diseño, montaje, ejecución y control de procesos logísticos, teniendo en cuenta que en todos estos problemas se requiere tomar decisiones óptimas o casi óptimas que involucran variables sujetas a requerimientos. Hay tres componentes básicas en el proceso de decisión que deben ser determinadas para formular un modelo de simulación, que son:

- Las variables de decisión

- Restricciones del problema

- Determinar la función objetivo a optimizar.

Después se debe elegir un método existente o desarrollar un nuevo algorit-

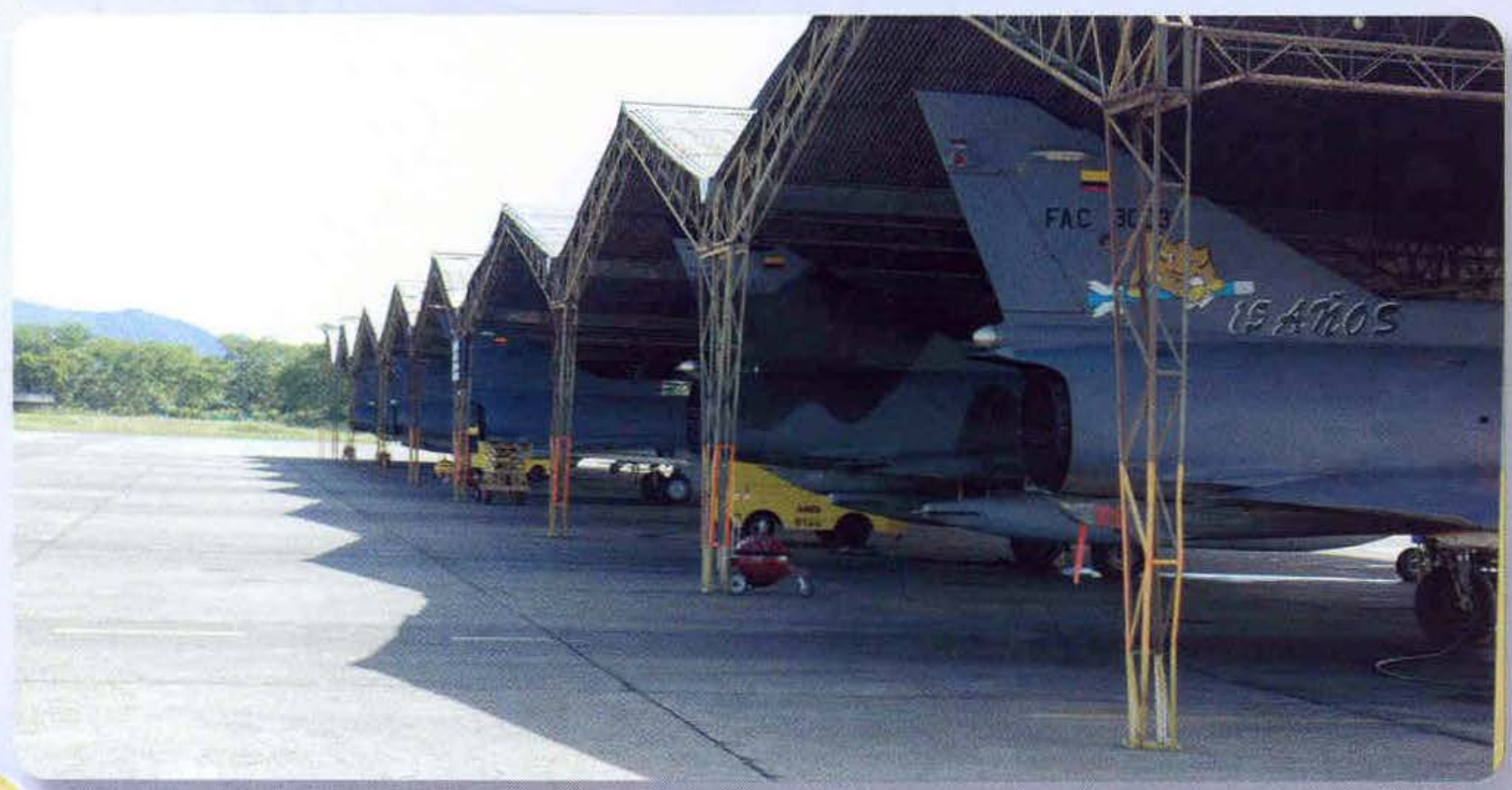


mo que encuentre la solución óptima o aproximada.

Usualmente, se relaciona la palabra simu lación con herramientas computacionales como videojuegos o realidad virtual, y en el caso de la Fuerza Aérea, con la palabra "juegos de guerra" ó "simulador de vue lo". Pero en el diseño y optimización de procesos logísticos, la simulación es defi nida como el proceso por el cual se cons truye, ejecuta y analiza un proceso o caso, siguiendo las variables y restricciones de la vida real. La ventaja de la utilización de herramientas como la simulación es que el sistema o modelo puede ser someti do a diferentes escenarios, y es posible analizar variables como la incertidumbre. Otra de las ventajas de la simulación es que permite el análisis de modelos y sis temas antes de ser implementados en la vida real, proceso que ahorra la inversión en recursos económicos, logísticos, tec nológicos y humanos, porque se puede llegar al resultado de aceptar o rechazar una posible hipótesis. Sin embargo, mu chos modelos, debido a su complejidad, requieren tiempo para diseñarlos y ejecu tarlos: además, el proceso de validación del modelo suele ser compleja en medida en que existe una mayor complejidad del sistema.

Para el diseño de un modelo de simula ción es necesario desarrollar los siguien tes pasos:

- Formular, definir e identificar el proble ma, a través del diseño de un modelo conceptual, basado en las variables y restricciones del sistema, que sea repre sentativo de los principales elementos de la vida real, teniendo en cuenta que un modelo no es una fiel fotocopia del caso real sino que refleja las caracterís ticas más relevantes del problema.

- Construir el modelo compu tacional, basado en los diferentes elementos definidos en el problema, teniendo en cuenta que cada lenguaje de simulación tiene definidas ciertas ca racterísticas, entre ellas tenemos como sigue:

Entidades: los elementos sometidos a la simulación, como por ejemplo pro ductos, documentos, estibas, mer cancía consolidada, entre otros.

Recursos: aquellos elementos que fa cilitan el proceso a simular, por ejem plo, operarios, equipos móviles de manejo de carga (estibadoras, mon tacargas, gruas, entre otros)

- Plazas productivas:máquinas, equi pos, puestos de trabajo, áreas que intervienen en el proceso de simula ción, por ejemplo, muelles de carga, áreas de almacenamiento, oficinas, líneas de producción, entre otros.

- Grafos: aquellas redes o caminos por los cuales se mueve un recurso de un área productiva a otra.

- Verificar y desarrollar e! modelo: este paso también conocido como el de depu ración y ejecución permite revisar la inte rrelación entre las variables, restricciones y elementos de entrada y los resultados que se esperan tras simular en tiempo real o de una forma acelerada la aplicación di señada.

- Recolección de información y análisis de resultados: es un proceso de valor agregado al proceso de simulación, en el cual se obienen conclusiones y reco 
mendaciones sobre la aplicación de un sistema productivo o logístico. Estos resultados son usualmente expresados en términos de:

- Estadísticas básicas sobre las entradas y salidas del modelo o Eficiencia en la utilización de recursos.

- Porcentaje de utilización, bloqueo e ineficiencia de los recursos utilizados.

- Costos y ganancias generadas por el modelo.

La simulación ha surgido como una herramienta para el diseño, administraron y opti- mización de sistemas logísticos, teniendo en cuenta que existe una mayor complejidad de los sistemas en las organizaciones militares y privadas

La simulación es una de las estrategias utilizadas con el fin de sincronizar los procesos logísticos con las necesidades, requerimientos y exigencias del cliente, encontrando un incremento de la razón beneficio costo. Los procesos logísticos requieren que métodos como la simulación identifiquen y evalúen el desarrollo, eficiencia, eficacia y por consiguiente la efectividad de las operaciones logísticas.

\section{BIBUIOGRAFIA}

April, I. y otros tres autores, New advances and applications for marrying simulation and optimization, Actas de 2004 Winter Simulation Conference, 1, 76-82, Washington DC, USA, 5 al 8 de Diciembre (2004).

Bandinelli, R. y otros tres autores, Using simulation for supply chain analysis: reviewing and proposing distributed simulation frameworks, Production Planning \& Control, 17(2), 167-175 (2006).

Ding, H. Y otros cuatro autores, "ONE" a new tool for Supply Chain Network optiftlization and simulation, Actas de 2004 Winter Simulation, 2, 343- 350, Washington DC, USA, 5 al 8 de Diciembre (2004).

Sarjoughian, H.S. y otros seis autores, Hybrid Discrete Event Simularian with Model Predictive Control for Semiconductor Supply-Chain Manufacturing, Actas de 2005 Winter Simulation Conference 256-266, Orlando, USA, 4 al 7 Diciembre (2005)

Umeda, S. y F. Zhang, Supply chain simularian: generic models and application examples, Production Planning \& Control, 17(2), 155-166 (2006).

Villegas, F. A. Y N. R. Smithz, Supply chain dynamics: analysis of inventory vs, arder oscillations tradeoff; Int. I. of Production Research, 44(6), 1037-1054 (2006).

Zhang, C. y otros tres autores, Sharing shipment quantity information in the supply chain, Omega 34 (5), $427-438$ (2006). Tambien en línea: httD:/MWW.SCODus.com/scoDus/search/form.url. Acceso: 12 de Junio (2006). 